\title{
A multicenter, open-label, single-arm phase I trial of neoadjuvant nivolumab monotherapy for resectable gastric cancer
}

\author{
Hirotaka Hasegawa ${ }^{1} \cdot$ Kohei Shitara $^{2} \cdot$ Shuji Takiguchi $^{3} \cdot$ Noriaki Takiguchi $^{4} \cdot$ Seiji Ito $^{5} \cdot$ Mitsugu Kochi $^{6}$. \\ Hidehito Horinouchi ${ }^{7} \cdot$ Takahiro Kinoshita $^{8} \cdot$ Takaki Yoshikawa $^{9} \cdot$ Kei Muro $^{10} \cdot$ Hiroyoshi Nishikawa $^{11,12}$. \\ Hideaki Suna $^{13} \cdot$ Yasuhiro Kodera $^{1}[$
}

Received: 8 November 2021 / Accepted: 9 February 2022 / Published online: 7 March 2022

(c) The Author(s) 2022

\begin{abstract}
Background Nivolumab monotherapy has demonstrated superior efficacy in advanced unresectable gastric cancer (GC), but its impact on resectable GC remains unknown. This phase I study aimed to evaluate safety, feasibility, and potential biomarkers of neoadjuvant nivolumab monotherapy in resectable GC.

Methods Untreated, resectable, cT2 or more advanced gastric adenocarcinomas with clinical stage I, II, or III were treated with two doses of nivolumab before gastrectomy. Patients were excluded if their tumors may be applicable to neoadjuvant chemotherapy. The primary endpoint was the incidence of adverse event (AE) categories of special interest.

Results All of the 31 enrolled patients completed 2 doses of nivolumab monotherapy. While 30 (97\%) patients underwent surgery with curative intent, 1 patient discontinued before the planned surgical intervention because of a newly emerging liver metastasis. Seven patients (23\%) had nivolumab treatment-related AEs, and one patient had a treatment-related AE of grade 3-4. The incidences of treatment-related AE categories of special interest ranged from 0 to $6 \%$. Notable surgical complications included two cases of grade 3 anastomotic leakage and two cases of pancreatic fistula. The major pathologic response (MPR) assessed by the independent pathology review committee was achieved in five (16\%) patients, of which one patient had a pathologic complete response. The MPR was mostly observed in patients with positive PD-L1 expression, high microsatellite instability, and/or high tumor mutation burden.

Conclusions Neoadjuvant nivolumab monotherapy is feasible with an acceptable safety profile and induces a MPR in certain patients with resectable GC. (Registration: clinicaltrials.jp, JapicCTI-183895).
\end{abstract}

Keywords Neoadjuvant therapy $\cdot$ Gastric cancer $\cdot$ Nivolumab $\cdot$ Biomarker $\cdot$ PD-L1

\section{Introduction}

Gastric cancer (GC) is one of the most common cancers in the world, with more than 1 million new incidences and approximately 770,000 deaths in 2020 [1]. Perioperative chemotherapy with triplet regimens in addition to surgery is the standard of care in the West [2-4]. Phase III evidence in this regard is yet to be generated in the Eastern hemisphere [5], where gastrectomy with lymphadenectomy followed

Hirotaka Hasegawa and Kohei Shitara contributed equally to this work.

Yasuhiro Kodera

ykodera@med.nagoya-u.ac.jp

Extended author information available on the last page of the article by postoperative chemotherapy remains the standard $[6,7]$. Nevertheless, Asian investigators have been keen to explore neoadjuvant chemotherapy, which has potential benefits in terms of early exposure of potential micrometastases to cytotoxic agents in addition to the likelihood of tumor shrinkage. These attempts have shown safety with a high compliance to the preoperative treatment in addition to down-staging and a high R0 resection rate in the neoadjuvant treatment arm [5].

Nivolumab is an immune checkpoint inhibitor of the programmed cell death 1 receptor (PD-1). Nivolumab monotherapy has demonstrated superior efficacy compared with placebo or standard chemotherapy in multiple types of cancer, including advanced GC, non-small-cell lung cancer (NSCLC), renal-cell carcinoma, and squamous-cell carcinoma of the head and neck [8-12]. However, although the efficacy of preoperative cytotoxic agents has been well 
documented in GC, the clinical and molecular effects of preoperative nivolumab monotherapy on GC tissue remain virtually unknown and are of considerable interest. Although the objective response rate of nivolumab monotherapy in heavily pretreated patients reaches $11.9 \%$ [13], the response to immunotherapy may not be negligible in relatively early stage GC; notably, nivolumab monotherapy demonstrated a pathologic response rate of up to $45 \%$ in resectable NSCLC $[14,15]$. In addition, the establishment of optimal biomarkers can justify the delivery of this relatively less toxic treatment for selected patients.

Accordingly, we conducted this phase I study to assess the safety and feasibility of neoadjuvant nivolumab monotherapy in patients with resectable GC. The correlations between conventional biomarkers and the clinical response were also evaluated.

\section{Methods}

\section{Study design}

This study is a part of the ONO-4538-67 study (JapicCTI-183895), which is a multicenter, open-label, singlearm phase I trial of neoadjuvant nivolumab monotherapy for resectable malignancies of GC or NSCLC in Japan. Patients with resectable GC received 2 doses of intravenous nivolumab at a dose of $240 \mathrm{mg} /$ body every 2 weeks, which is the same as the previous trial for NSCLC [14]. Gastrectomy with curative intent was scheduled at least 14 days after the last nivolumab dose. Thus, the duration of neoadjuvant therapy, including a 2 -week interval before surgery, was designed to be as short as 4 weeks, given that the response rate of nivolumab monotherapy in heavily pretreated GC had been modest compared with optimal combinations of cytotoxic agents currently used in the neoadjuvant setting.

The primary endpoint was the incidence of adverse event (AE) categories of special interest, including endocrinopathies, gastrointestinal toxicities, hepatotoxicities, pulmonary toxicities, renal toxicities, dermatologic toxicities, and infusion reactions. The secondary endpoints were any AEs and efficacy, which included major pathologic response (MPR) rate, pathologic complete response ( $\mathrm{pCR}$ ) rate, shrinkage of the primary tumor assessed by endoscopy, and $\mathrm{R} 0$ resection rate. The other key endpoints were immunologic, genomic, and pathological correlations of responses in blood and tumor samples acquired by pretreatment endoscopic biopsy and surgery.

The study protocol was approved by the institutional review board or independent ethic committee at each study site. This study follows the Good Clinical Practice guidelines of International Council for Harmonisation. All patients provided a written informed consent in accordance with the Declaration of Helsinki.

\section{Patients}

Patients were eligible for enrolment if they had untreated, resectable, cT2 or more advanced, histopathologically confirmed gastric adenocarcinoma with clinical stage I, II, or III, as indicated in the TNM Classification of Malignant Tumours (8th edition) of the Union for International Cancer Control [16]; were 20 years old or older; had Eastern Cooperative Oncology Group performance status (ECOG PS) of 0 or 1; had adequate organ function. Given the modest response rate of nivolumab monotherapy observed in heavily pretreated GC, patients were excluded if their tumor may be applicable to neoadjuvant chemotherapy, such as those with bulky lymph node (two or more adjacent lymph nodes of $1.5 \mathrm{~cm}$ diameter or a $3 \mathrm{~cm}$ diameter cluster of lymph nodes along the celiac, splenic, common hepatic, or proper hepatic arteries or the superior mesenteric vein) detected by contrast-enhanced abdominal computed tomography and linitis plastica-type GC $[5,17]$. Cancer of the gastric remnant was also excluded. Other key exclusion criteria were locally advanced unresectable or metastatic tumors, severe malnutrition, and active autoimmune disorders. Only patients with an ECOG PS of 0 or 1, without major relevant clinical findings that may increase the risk of surgery to an unacceptable level, without disease progression in the form of distant metastasis, and with expected $\mathrm{R} 0$ resection were indicated for subsequent surgery. The administration of immunosuppressant, corticosteroids equivalent to $>10 \mathrm{mg} /$ day prednisone, and other anticancer therapy were prohibited during the study period.

\section{Assessments}

Patient characteristics, including laboratory values, were assessed upon the enrolment, periodically during the treatment and perioperative periods, and at 30 days after the surgery or 60 days after the last nivolumab dose, whichever was later.

We assessed the AEs occurring from the first nivolumab dose until 30 days after the surgery or 60 days after the last nivolumab dose, whichever was later, or until the time of study discontinuation. Intraoperative and postoperative complications were assessed until 30 days after surgery. Each AE was graded in accordance with the National Cancer Institute Common Terminology Criteria for Adverse Events version 4.0 .

Tumors were assessed by computed tomography and magnetic resonance imaging of the chest, abdomen, and pelvis and by upper gastrointestinal endoscopy at the enrolment and just before surgery in accordance with the 
Response Evaluation Criteria in Solid Tumors (RECIST) guidelines (version 1.1). Responses in the primary tumor were assessed by upper gastrointestinal endoscopy and classified as follows: endoscopic complete response (eCR) if no tumor was observed; endoscopic partial response (ePR) if remarkable regression of the tumor was observed $(\leq 2 / 3$ in the diameter, $\leq 1 / 2$ in the area, and $\leq 1 / 3$ in the volume); endoscopic progressive disease (ePD) if the tumor was evidently enlarged; endoscopic stable disease (eSD) in other cases [18].

Surgical specimens, including primary gastric tumor and lymph nodes, were staged in accordance with the Japanese Classification of Gastric Carcinoma [16, 18]. The independent pathology review committee (IPRC) pathologically assessed the percentage of residual viable tumor that was identified on surgically resected specimens, which were stained routinely with hematoxylin and eosin, and tumors with no more than $10 \%$ viable tumor cells were considered to have had a MPR [19].

Tumor proportion score (TPS; the proportion of programmed cell death-ligand 1 (PD-L1)-positive cells among tumor cells) and combined positive score (CPS; the ratio of the sum of PD-L1-positive tumor cells, lymphocytes, and macrophages to the number of all tumor cells) were centrally assessed using archival tumor tissues with the PD-L1 IHC 28-8 pharmDx kit (Agilent Technologies Inc. Santa Clara, CA, USA). Tumor mutation burden (TMB) and microsatellite instability (MSI) were also assessed at the central laboratory using FoundationOne (Foundation Medicine Inc, Cambridge, MA, USA) and the MSI Analysis System (Promega Corp, Madison, WI, USA), respectively. Tumors with $\geq 10$ mutations $/ \mathrm{Mb}$ and with $<10$ mutations/Mb were defined as TMB-High and TMB-Low, respectively.

\section{Statistics}

The planned number of patients was 30 . The average incidence of the $\mathrm{AE}$ categories of special interest was $10.8 \%$ in patients with unresectable advanced or metastatic GC that was refractory or intolerant to standard chemotherapy in the phase III ATTRACTION-2 study [8]. The incidence of AE categories of special interest was considered lower in this study than that in ATTRACTION-2 because only two doses were administered in this study. When the true incidence of the AE categories of special interest in this neoadjuvant nivolumab monotherapy was postulated as $3 \%, 5 \%$, and $7 \%$, at least 1 among the 30 patients might have experienced these AEs with a probability of $59.9 \%, 78.5 \%$, and $88.6 \%$, respectively. Thus, 30 patients may be sufficient to assess the AEs of special interest.

Safety was assessed in patients who received at least one dose of nivolumab. The efficacy was assessed in patients fulfilling the major eligible criteria. The pathologic response evaluable set constituted patients who underwent radical resection. The 95\% confidence interval for MPR rates was estimated by the Clopper-Pearson method.

\section{Data availability}

Qualified researchers may request Ono Pharmaceutical Co., Ltd. to disclose individual patient-level data from clinical studies through the following website: https://www.clinicalst udydatarequest.com/. For more information on the policy of Ono Pharmaceutical Co., Ltd. for the Disclosure of Clinical Study Data, please visit https://www.ono.co.jp/eng/rd/ policy.html.

\section{Results}

\section{Patients}

Between November 2018 and December 2019, 31 patients were enrolled at 7 study sites. Most of the patients were male (68\%) and had an ECOG PS score of 0 (97\%) (Table 1). The primary tumor sites were the stomach in $97 \%$ of patients and the esophagogastric junction in 3\% of patients (Online Resource 1). Lymph node metastasis was observed in $32 \%$ of patients, and most patients (68\%) were clinically confined to stages I or II. Table 1 and Online Resource 1 summarize the other clinical characteristics of the tumors.

\section{Safety and feasibility}

All of the 31 patients completed 2 doses of neoadjuvant nivolumab monotherapy; no AEs led to the discontinuation of nivolumab. AEs were observed in 21 (68\%) patients, of which 9 (29\%) were grade 3-4 AEs. Seven patients (23\%) had nivolumab treatment-related AEs, and one patient (3\%) had a treatment-related AE of grade 3-4, which was the grade 3 asymptomatic lipase increased. The most common AEs of special interest were alanine aminotransferase increased in four patients, aspartate aminotransferase increased in three, diarrhea in three, and rash in three (Table 2). Two patients had rash (grade 1 and 2) as nivolumab treatment-related AEs. Any AEs that have not been previously reported to be associated with nivolumab were not observed.

Among the 30 patients who underwent surgery, 15 (50\%) patients had intraoperative and postoperative complications of any grade (Online Resource 2). Notable surgical complications included two cases of grade 3 anastomotic leakage and two cases of pancreatic fistula. No intra-abdominal abscess was observed. 
Table 1 Patient baseline characteristics

\begin{tabular}{|c|c|}
\hline Characteristics & Nivolumab $(N=31)$ \\
\hline Median age-years (range) & $69(44-84)$ \\
\hline \multicolumn{2}{|l|}{$\operatorname{Sex}-n(\%)$} \\
\hline Male & $21(68)$ \\
\hline Female & $10(32)$ \\
\hline \multicolumn{2}{|l|}{ ECOG PS $-n(\%)$} \\
\hline 0 & $30(97)$ \\
\hline 1 & $1(3)$ \\
\hline \multicolumn{2}{|l|}{$\mathrm{T}$ classification- $n(\%)$} \\
\hline $\mathrm{T} 2$ & $7(23)$ \\
\hline $\mathrm{T} 3$ & $22(71)$ \\
\hline $\mathrm{T} 4 \mathrm{a}$ & $2(6)$ \\
\hline $\mathrm{T} 4 \mathrm{~b}$ & 0 \\
\hline \multicolumn{2}{|l|}{$\mathrm{N}$ classification $-n(\%)$} \\
\hline N0 & $21(68)$ \\
\hline N1 & $7(23)$ \\
\hline $\mathrm{N} 2$ & $3(10)$ \\
\hline \multicolumn{2}{|l|}{ M classification- $n(\%)$} \\
\hline M0 & $31(100)$ \\
\hline M1 & 0 \\
\hline \multicolumn{2}{|l|}{ Clinical stage $-n(\%)$} \\
\hline I & $7(23)$ \\
\hline IIA & 0 \\
\hline IIB & $14(45)$ \\
\hline III & $10(32)$ \\
\hline \multicolumn{2}{|l|}{ PD-L1 TPS— $n(\%)$} \\
\hline$<1 \%$ & $22(71)$ \\
\hline$\geq 1$ to $<10 \%$ & $6(19)$ \\
\hline$\geq 10 \%$ & $3(10)$ \\
\hline \multicolumn{2}{|l|}{ PD-L1 CPS- $n(\%)$} \\
\hline$<1$ & $11(35)$ \\
\hline$\geq 1$ to $<10$ & $11(35)$ \\
\hline$\geq 10$ & $9(29)$ \\
\hline \multicolumn{2}{|l|}{ MSI status- $n(\%)$} \\
\hline MSI-high & $7(23)$ \\
\hline MSI-low & $4(13)$ \\
\hline MSS & $20(65)$ \\
\hline \multicolumn{2}{|l|}{$\mathrm{TMB}-n(\%)$} \\
\hline High & $9(29)$ \\
\hline Low & 12 (39) \\
\hline Missing $^{\mathrm{a}}$ & $10(32)$ \\
\hline
\end{tabular}

$C P S$ combined positive score, ECOG PS Eastern Cooperative Oncology Group performance status, $M b$ mega base pairs, $M S I$ microsatellite instability, $M S S$ microsatellite stable, $T M B$ tumor mutation burden, TPS tumor proportion score

${ }^{a}$ Including those that were not evaluable and not determined

\section{Clinical efficacy}

The proportions of patients with ePR and eSD were 13\% and $84 \%$, respectively (Online Resource 3 ). No patient with target lesions in accordance with the RECIST criteria was observed. Meanwhile, 30 (97\%) patients underwent surgery with curative intent, and one (3\%) patient who had a T4a tumor discontinued after completing the nivolumab treatment before the planned surgical intervention because of liver metastasis that emerged as a new lesion. One patient underwent surgery behind the schedule due to grade 2 rash. R0 resection was achieved in 27 patients (90\% of the patients who underwent surgery with curative intent), whereas the remaining 3 patients had a macroscopic residual tumor (R2) resection due to peritoneal metastases that were undetected prior to enrolment.

\section{Pathological findings}

Online Resource 4 depicts the tumor characteristics upon the enrolment (clinical assessments) and after surgery (pathological assessment). Pathological T2 or lower was observed in 15 patients (48\%). The proportion of pathological stage $0 / \mathrm{I}$ patients was as high as $45 \%$, whereas that of clinical stage I patients upon enrolment was $23 \%$. On the other hand, no evident decrease in the proportion of $\geq$ stage III patients was observed. Although distant metastases had not been detected through imaging studies at the time of enrolment, one patient developed liver metastasis during the neoadjuvant immunotherapy, and three other patients had peritoneal metastasis that was recognized at surgery but not by imaging just before the surgery; no staging laparoscopy, when conducted, found peritoneal metastasis before enrolment.

All of the 30 patients who underwent surgery provided specimens with diverse levels of tumor regression (Fig. 1). An IPRC-assessed MPR in the primary tumor was achieved in five (16\%) patients, of which one had pCR. The patient who achieved pCR had a clinical stage I disease, whereas other cases of MPR were observed among patients with clinical stages IIB and III (Online Resource 5). Three out of five patients with MPR had ePR, and the other two, including one with pCR, had eSD. The majority of the patients with a MPR were those with $\geq 1 \%$ PD-L1 TPS (80\%), $\geq 10$ PD-L1 CPS (80\%), MSI-High (80\%), and TMB-High (60\%) (Fig. 2). Among the patients without MPR, the proportions of patients with MSI-High and TMB-High were $12 \%$ and $20 \%$, respectively (Online Resource 6).

Fibrosis without apparent tumor cells was observed in the surgically resected specimens of the patient who achieved pCR (Online Resource 7).

\section{Discussion}

Neoadjuvant therapy with two doses of nivolumab in patients with GC was safe and had no new safety signal. Considering relatively high rate of disease progression 
Table 2 AEs

\begin{tabular}{|c|c|c|c|c|}
\hline \multirow[t]{2}{*}{$N=31$} & \multicolumn{2}{|l|}{ AEs } & \multicolumn{2}{|c|}{ Treatment-related AEs } \\
\hline & Any grade & Grade 3-4 & Any grade & Grade 3-4 \\
\hline Any & $21(68)$ & $9(29)$ & $7(23)$ & $1(3)$ \\
\hline \multicolumn{5}{|l|}{ AEs of special interest } \\
\hline ALT increased & $4(13)$ & $2(6)$ & $1(3)$ & 0 \\
\hline AST increased & $3(10)$ & $1(3)$ & 0 & 0 \\
\hline Blood creatinine increased & $1(3)$ & 0 & 0 & 0 \\
\hline Dermatitis & $1(3)$ & 0 & 0 & 0 \\
\hline Diarrhea & $3(10)$ & 0 & $1(3)$ & 0 \\
\hline Eczema & $1(3)$ & 0 & 0 & 0 \\
\hline GGT increased & $2(6)$ & $2(6)$ & 0 & 0 \\
\hline Hypothyroidism & $1(3)$ & 0 & $1(3)$ & 0 \\
\hline Rash & $3(10)$ & 0 & $2(6)$ & 0 \\
\hline \multicolumn{5}{|l|}{ Treatment-related AEs } \\
\hline ALT increased & $4(13)$ & $2(6)$ & $1(3)$ & 0 \\
\hline Cardiomyopathy & $1(3)$ & 0 & $1(3)$ & 0 \\
\hline Diarrhea & $3(10)$ & 0 & $1(3)$ & 0 \\
\hline Fatigue & $1(3)$ & 0 & $1(3)$ & 0 \\
\hline Hypothyroidism & $1(3)$ & 0 & $1(3)$ & 0 \\
\hline Lipase increased & $1(3)$ & $1(3)$ & $1(3)$ & $1(3)$ \\
\hline Pancreatic fistula & $2(6)$ & 0 & $1(3)$ & 0 \\
\hline Rash & $3(10)$ & 0 & $2(6)$ & 0 \\
\hline Vomiting & $1(3)$ & 0 & $1(3)$ & 0 \\
\hline
\end{tabular}

We assessed the AEs that occurred from the first nivolumab dose until 30 days after the surgery or 60 days after the last nivolumab dose, whichever was later, or until the time of study discontinuation. The number $(\%)$ of patients is shown. Nivolumab treatment-related AEs are shown on the right

$A E$ adverse event, $A L T$ alanine aminotransferase, $A S T$ aspartate aminotransferase, GGT gamma-glutamyltransferase

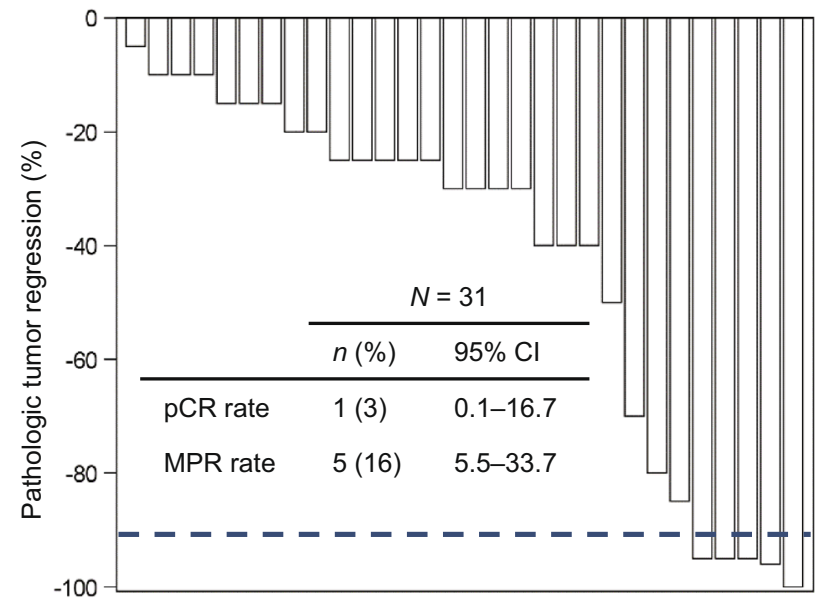

Fig. 1 Pathologic response. The waterfall plot shows the tumor reduction ratio calculated from residual tumors in the resected specimens. The tumor reduction in the patient who did not undergo surgery was not evaluated. The dotted line indicates $90 \%$ reduction. $C I$ confidence interval, $M P R$ major pathologic response, $p C R$ pathologic complete response after nivolumab for metastatic GC in the ATTRACTION-2 study [8], the number of the neoadjuvant treatment course had to be kept to minimal so as not to cause excessive delay in the surgical treatment. Because no prior information was available regarding efficacy of nivolumab monotherapy for resectable GC, we referred to the favorable results in the previous trial for NSCLC [14] and determined the duration of neoadjuvant treatment to be relatively short at 4 weeks. Nevertheless, two doses of nivolumab led to a MPR in $16 \%$ of patients who underwent surgery with curative intent. The R0 resection rate was favorable at $90 \%$.

Meanwhile, more than $80 \%$ of the patients treated with neoadjuvant chemotherapy for GC reported treatment-related AEs [3, 20, 21]; the neoadjuvant nivolumab monotherapy in this study had a lower incidence of treatment-related AEs at 23\%, which was comparable with the neoadjuvant nivolumab monotherapy for lung cancer (23\%) [14]. The incidences of treatment-related AEs of special interest ranged from 0 to $6 \%$, which were favorable compared with those observed during nivolumab monotherapy in the salvage line for advanced GC [8], although a direct 
Fig. 2 Major pathologic response rates. $C I$ confidence interval, $C P S$ combined positive score, $M S I$ microsatellite instability, MSS microsatellite stable, $N D$ not determined, $T M B$ tumor mutational burden, TPS tumor proportion score

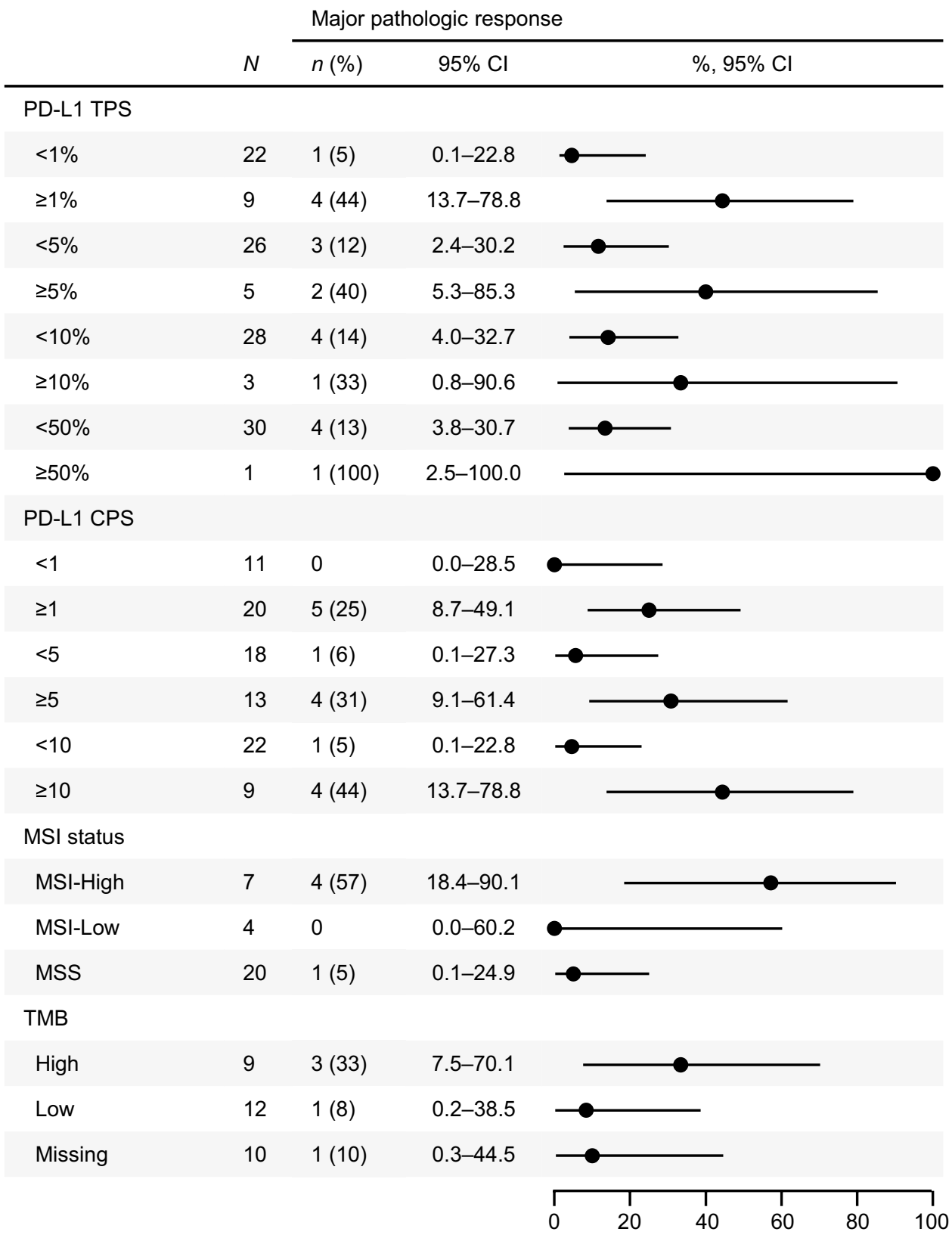

comparison may be inappropriate given that only two doses of nivolumab were administered in this study.

Although one patient (3\%) failed to undergo surgery due to a new lesion, the other patients $(97 \%)$ were subsequently treated with surgery, in which the $\mathrm{R} 0$ resection rate was $90 \%$. Five patients achieved a MPR, including one $\mathrm{pCR}$, and the number of patients with pathologically stage I disease was twice the number of patients who had clinically stage I disease at the initial presentation, suggesting the promising efficacy of neoadjuvant nivolumab monotherapy. In the FLOT4 study, the representative neoadjuvant chemotherapy achieved a $94 \%$ gastrectomy rate; however, $2 \%$ of patients suffered from disease progression or died during the neoadjuvant chemotherapy [3]. Other studies evaluating neoadjuvant chemotherapy for GC also demonstrated comparable gastrectomy and disease progression rates $[20,21]$. The MPR rate in neoadjuvant nivolumab monotherapy (16\%) was also comparable with those $(6 \%-32 \%)$ in patients with GC receiving neoadjuvant chemotherapy [4, 20-22]. However, such comparisons should be interpreted with caution, given that patients enrolled in the current study had GC at relatively early clinical stages. Although the planned treatment duration was designed to be relatively short at 4 weeks, at least one patient had a new lesion prior to surgery, and 3 other patients failed to undergo $\mathrm{R} 0$ resection due to macroscopic 
metastases that were inevident at the time of enrolment. We plan to seek for effect-predictive biomarkers in the forthcoming molecular analyses using clinical specimens collected before and after the nivolumab monotherapy.

Regarding several potential biomarkers that have already been evaluated, nivolumab and pembrolizumab monotherapies for pretreated or treatment-naïve GC have demonstrated relatively high response rates in patients with MSI [23-25]. A similar trend was observed in other tumors, including gastroesophageal cancer, colorectal cancer, and lung cancer [26-30]. Consistent with these reports, a high MPR (57\%) was observed in MSI-High tumors after two doses of nivolumab monotherapy in the neoadjuvant setting compared with those $(0-11 \%)$ observed in perioperative chemotherapy [31-33]. Given that cytotoxic agents remain the current standard perioperative therapy for resectable GC despite relatively poor efficacy for MSI-High tumors, this study may open the key for the future trial of neoadjuvant nivolumab monotherapy for resectable MSI-High GC. On the other hand, GC tumors with MSI-Low or microsatellite stable (MSS) responded well to perioperative chemotherapy [31-34], but poorly to the neoadjuvant nivolumab monotherapy. Mechanisms underlying the low response in MSI-Low and MSS remain to be addressed. One MSS tumor achieved a MPR, in which the PD-L1 TPS and CPS scores were 5\% and 15, respectively, and the TMB status was low, suggesting that the relatively high PD-L1 expression level might have contributed to the response. However, the sample size was too small to assess the correlation between the PD-L1 expression levels and response to neoadjuvant nivolumab monotherapy. Pembrolizumab monotherapy at the salvage line for advanced GC demonstrated higher benefits in patients with PD-L1-positive than in patients with PD-L1-negative [35], whereas the ATTRACTION-2 study demonstrated the benefit of nivolumab monotherapy regardless of the PD-L1 expression levels although PD-L1 TPS was determined only in a limited number of patients [13]. The benefit of neoadjuvant nivolumab monotherapy on patients with lung cancer was obtained regardless of the PD-L1 expression levels [14]. The controversial issue regarding response to immunotherapy and PD-L1 expression status will be addressed in the future. Furthermore, the resected specimens obtained in this study were valuable in ascertaining the immune effector function of nivolumab within the GC microenvironment during the therapeutic time window. In the meantime, a combination of perioperative chemotherapy with immunotherapy may be promising, as shown in untreated, unresectable GC in CheckMate 649 and ATTRACTION-4 studies, and therefore has been evaluated for resectable GC in ongoing phase III clinical trials, including ATTRACTION-5 study for nivolumab, KEYNOTE-585 study for pembrolizumab, and MATTERHORN study for durvalumab.
The small number of patients limited the interpretation of the results, especially in the subpopulation analysis. Other limitations of this study include the absence of a comparator group, lack of ethnic diversity, and relatively early stages of tumors. Long-term follow-up is required to assess the advantages of neoadjuvant nivolumab monotherapy, given that MPR only serves as a surrogate of survival endpoints.

In conclusion, neoadjuvant nivolumab monotherapy is feasible with an acceptable safety profile, and it induces a MPR in certain patients with resectable GC despite the short duration. Further molecular analyses for the identification of predictive markers of the response are awaited to establish an optimal neoadjuvant therapy with nivolumab, either alone or in combination with cytotoxic agents.

Supplementary Information The online version contains supplementary material available at https://doi.org/10.1007/s10120-022-01286-w.

Acknowledgments We thank the patients who participated and their supportive families for making this study possible. We also thank medical professionals in the study sites. We are grateful to the IPRC members, including Dr. Takeshi Kuwata (National Cancer Center Hospital East) and Dr. Hiroshi Kawachi (Cancer Institute Hospital of Japanese Foundation for Cancer Research). A medical written support was provided by Masatoshi Esaki, PhD, of Ono Pharmaceutical, Co., Ltd.

Funding This study was funded by Ono Pharmaceutical Co., Ltd. and Bristol Myers Squibb Company.

\section{Declarations}

Conflict of interest H. Hasegawa reports grants from Ono Pharma, grants from Bristol Myers Squibb, during the conduct of the study. KS reports grants and personal fees from Ono Pharma, grants and personal fees from Bristol Myers Squibb, during the conduct of the study; grants and personal fees from Astellas, grants and personal fees from Eli Lilly, grants from Sumitomo Dainippon, grants from Daiichi Sankyo, grants and personal fees from Taiho Pharmaceutical, grants from Chugai Pharmaceutical, grants and personal fees from MSD, grants from Medi Science, personal fees from Takeda Pharmaceutical, personal fees from Pfizer, personal fees from Novartis, personal fees from Abbvie, personal fees from GSK, personal fees from Yakult, outside the submitted work. ST reports grants from Ono Pharma, grants from Bristol Myers Squibb, during the conduct of the study. NT reports grants from Ono Pharma, grants from Bristol Myers Squibb, during the conduct of the study. SI reports grants from Ono Pharma, grants from Bristol Myers Squibb, during the conduct of the study; grants from MSD, grants and personal fees from AstraZeneca, personal fees from Taiho Pharmaceutical, outside the submitted work. MK reports grants from Ono Pharma, grants from Bristol Myers Squibb, during the conduct of the study. H Horinouchi reports grants from Ono Pharma, grants and personal fees from Bristol Myers Squibb, during the conduct of the study; grants from Taiho Pharmaceutical, grants from Merck Serono, grants and personal fees from Novartis, grants and personal fees from MSD, grants and personal fees from Chugai Pharmaceutical, grants from Genomic Health, grants from A2 Healthcare, grants from Daiichi Sankyo, grants from AbbVie, personal fees from Eli Lilly, personal fees from AstraZeneca, personal fees from Kyowa Kirin, outside the submitted work. TK reports grants and personal fees from Ono Pharma, grants and personal fees from Bristol Myers Squibb, during the conduct of the study. TY reports grants and per- 
sonal fees from Ono Pharma, grants and personal fees from Bristol Myers Squibb, during the conduct of the study; grants from Eli Lilly, personal fees from Daiichi Sankyo, personal fees from MSD, personal fees from Taiho Pharmaceutical, personal fees from Terumo, personal fees from Chugai Pharmaceutical, personal fees from Miyarisan, personal fees from EA Pharmaceutical, personal fees from AstraZeneca, personal fees from Otsuka Pharmaceutical, personal fees from Johnson and Johnson, personal fees from Covidien, outside the submitted work. KM reports grants and personal fees from Ono Pharma, grants and personal fees from Bristol Myers Squibb, during the conduct of the study; grants from Solasia Pharmaceutical, grants from Merck Serono, grants from Daiichi Sankyo, grants from Parexel International, grants from Pfizer, grants from MSD, grants and personal fees from Amgen, grants and personal fees from Sanofi, grants and personal fees from Taiho Pharmaceutical, personal fees from AstraZeneca, personal fees from Chugai Pharmaceutical, personal fees from Takeda, personal fees from Eli Lilly, personal fees from Bayer, outside the submitted work. $\mathrm{HN}$ reports grants and personal fees from Ono Pharma, grants and personal fees from Bristol Myers Squibb, during the conduct of the study; grants and personal fees from Chugai Pharmaceutical, grants and personal fees from MSD, grants from Taiho Pharmaceutical, grants from Daiichi Sankyo, grants from Kyowa Kirin, grants from Zenyaku Kogyo, grants from Oncollys Biopharma, grants from Deviopharma, grants from Asahi-Kasei, grants from Sysmex, grants from Fijifilm, grants from SRL, grants from Astellas, grants from Sumitomo Dainippon, grants from BD Japan, outside the submitted work. HS reports other from Ono Pharma, during the conduct of the study. YK reports grants from Ono Pharma, grants from Bristol Myers Squibb, during the conduct of the study; grants from Kaken Pharma, grants from Covidien, grants from EA Pharma, grants from Novartis, grants from KCI, grants from Maruho, grants and personal fees from Daiichi Sankyo, grants from Otsuka, grants and personal fees from Tsumura, grants from Sawai, grants and personal fees from Taiho Pharma, grants and personal fees from Chugai Pharma, grants and personal fees from Eli Lilly, grants and personal fees from Johnson \& Johnson, grants and personal fees from Takeda, grants and personal fees from Yakult, grants and personal fees from Otsuka, grants and personal fees from Ono Pharma, grants and personal fees from Covidien, grants and personal fees from MSD, grants from Sanofi, grants from Shionogi, grants from Nihon Kayaku, personal fees from Bristol Myers Squibb, outside the submitted work.

Ethical standards All procedures followed the ethical standards of the responsible committee on human experimentation (institutional and national) and the Helsinki Declaration of 1964 and later versions.

Informed consent Informed consent to be included in this study was obtained from all patients.

Open Access This article is licensed under a Creative Commons Attribution 4.0 International License, which permits use, sharing, adaptation, distribution and reproduction in any medium or format, as long as you give appropriate credit to the original author(s) and the source, provide a link to the Creative Commons licence, and indicate if changes were made. The images or other third party material in this article are included in the article's Creative Commons licence, unless indicated otherwise in a credit line to the material. If material is not included in the article's Creative Commons licence and your intended use is not permitted by statutory regulation or exceeds the permitted use, you will need to obtain permission directly from the copyright holder. To view a copy of this licence, visit http://creativecommons.org/licenses/by/4.0/.

\section{References}

1. Sung H, Ferlay J, Siegel RL, Laversanne M, Soerjomataram I, Jemal A, et al. Global cancer statistics 2020: GLOBOCAN estimates of incidence and mortality worldwide for 36 cancers in 185 countries. CA Cancer J Clin. 2021;71:209-49.

2. Cunningham D, Allum WH, Stenning SP, Thompson JN, Van de Velde CJH, Nicolson M, et al. Perioperative chemotherapy versus surgery alone for resectable gastroesophageal cancer. N Engl J Med. 2006;355:11-20.

3. Al-Batran SE, Homann N, Pauligk C, Goetze TO, Meiler J, Kasper $\mathrm{S}$, et al. Perioperative chemotherapy with fluorouracil plus leucovorin, oxaliplatin, and docetaxel versus fluorouracil or capecitabine plus cisplatin and epirubicin for locally advanced, resectable gastric or gastro-oesophageal junction adenocarcinoma (FLOT4): a radomised, phase 2/3 trial. Lancet. 2019;393:1948-57.

4. Al-Batran SE, Hofheinz RD, Pauligk C, Kopp HG, Haag GM, Luley $\mathrm{KB}$, et al. Histopathological regression after neoadjuvant docetaxel, oxaliplatin, fluorouracil, and leucovorin versus epirubicin, cisplatin, and fluorouracil or capecitabine in patients with resectable gastric or gastro-oesophageal junction adenocarcinoma (FLOT4-AIO): results from the phase 2 part of a multicentre, open-label, randomised phase $2 / 3$ trial. Lancet Oncol. 2016;17:1697-708.

5. Iwasaki Y, Terashima M, Mizusawa J, Katayama H, Nakamura K, Katai $\mathrm{H}$, et al. Gastrectomy with or without neoadjuvant $\mathrm{S}-1$ plus cisplatin for type 4 or large type 3 gastric cancer (JCOG0501): an open-label, phase 3, randomized controlled trial. Gastric Cancer. 2021;24:492-502.

6. Noh SH, Park SR, Yang HK, Chung HC, Chung IJ, Kim SW, et al. Adjuvant capecitabine plus oxaliplatin for gastric cancer after D2 gastrectomy (CLASSIC): 5-year follow-up of an open-label, randomised phase 3 trial. Lancet Oncol. 2014;15:1389-96.

7. Sasako M, Sakuramoto S, Katai H, Kinoshita T, Furukawa $\mathrm{H}$, Yamaguchi $\mathrm{T}$, et al. Five-year outcomes of a randomized phase III trial comparing adjuvant chemotherapy with S-1 versus surgery alone in stage II or III gastric cancer. J Clin Oncol. 2011;29:4387-93.

8. Kang Y-K, Boku N, Satoh T, Ryu MH, Chao Y, Kato K, et al. Nivolumab in patients with advanced gastric or gastro-oesophageal junction cancer refractory to, or intolerant of, at least two previous chemotherapy regimens (ONO-4538-12, ATTRAC TION-2): a randomised, double-blind, placebo-controlled, phase 3 trial. Lancet. 2017;390:2461-71.

9. Motzer RJ, Escudier B, McDermott DF, George S, Hammers HJ, Srinivas $\mathrm{S}$, et al. Nivolumab versus everolimus in advanced renalcell carcinoma. N Engl J Med. 2015;373:1803-13.

10. Ferris RL, Blumenschein G, Fayette J, Guigay J, Colevas AD, Licitra L, et al. Nivolumab for recurrent squamous-cell carcinoma of the head and neck. N Engl J Med. 2016;375:1856-67.

11. Borghaei H, Paz-Ares L, Horn L, Spigel DR, Steins M, Ready NE, et al. Nivolumab versus docetaxel in advanced nonsquamous non-small-cell lung cancer. N Engl J Med. 2015;373:1627-39.

12. Brahmer J, Reckamp KL, Baas P, Crinò L, Eberhardt WEE, Poddubskaya E, et al. Nivolumab versus docetaxel in advanced squamous-cell non-small-cell lung cancer. N Engl J Med. 2015;373:123-35.

13. Chen L-T, Satoh T, Ryu MH, Chao Y, Kato K, Chung HC, et al. A phase 3 study of nivolumab in previously treated advanced gastric or gastroesophageal junction cancer (ATTRACTION-2): 2-year update data. Gastric Cancer. 2020;23:510-9.

14. Forde PM, Chaft JE, Smith KN, Anagnostou V, Cottrell TR, Hellmann MD, et al. Neoadjuvant PD-1 blockade in resectable lung cancer. N Engl J Med. 2018;378:1976-86. 
15. Cascone T, William WN, Weissferdt A, Leung CH, Lin HY, Pataer A, et al. Neoadjuvant nivolumab or nivolumab plus ipilimumab in operable non-small cell lung cancer: the phase 2 randomized NEOSTAR trial. Nat Med. 2021;27:504-14.

16. Brierley JD, Gospodarowicz MK, Wittekind C. TNM Classification of Malignant Tumours, 8th Edition: Wiley-Blackwell, 2016.

17. Ito S, Sano T, Mizusawa J, Takahari D, Katayama H, Katai $\mathrm{H}$, et al. A phase II study of preoperative chemotherapy with docetaxel, cisplatin, and S-1 followed by gastrectomy with D2 plus para-aortic lymph node dissection for gastric cancer with extensive lymph node metastasis: JCOG1002. Gastric Cancer. 2017;20:322-31.

18. Japanese Gastric Cancer Association. Japanese classification of gastric carcinoma: 15th edition. Tokyo: Kanehara Shuppan; 2017.

19. Becker K, Mueller JD, Schulmacher C, Ott K, Fink F, Busch $\mathrm{R}$, et al. Histomorphology and grading of regression in gastric carcinoma treated with neoadjuvant chemotherapy. Cancer. 2003;98:1521-30.

20. Berenato R, Morano F, Pietrantonio F, Cotsoglou C, Caporale $\mathrm{M}$, Infante G, et al. Preoperative capecitabine, oxaliplatin, and irinotecan in resectable gastric or gastroesophageal junction cancer: Pathological response as primary endpoint and FDG-PET predictions. Oncology. 2017;93:279-86.

21. Sah BK, Zhang B, Zhang H, Li J, Yuan F, Ma T, et al. Neoadjuvant FLOT versus SOX phase II randomized clinical trial for patients with locally advanced gastric cancer. Nat Commun. 2020;11:6093.

22. Petrioli R, Marrelli D, Roviello F, D'Ignazio A, Torre P, Chirra $\mathrm{M}$, et al. Pathological response and outcome after neoadjuvant chemotherapy with DOC (docetaxel, oxaliplatin, capecitabine) or EOF (epirubicin, oxaliplatin, 5-fluorouracil) for clinical T3-T4 non-metastatic gastric cancer. Surg Oncol. 2020;32:2-7.

23. Petrillo A, Tirino G, Marino FZ, Pompella L, Sabetta R, Panarese $\mathrm{I}$, et al. Nivolumab in heavily pretreated metastatic gastric cancer patients: real-life data from a western population. Onco Targets Ther. 2020;13:867-76.

24. Shitara K, Van Cutsem E, Bang YJ, Fuchs C, Wyrwicz L, Lee $\mathrm{KW}$, et al. Efficacy and safety of pembrolizumab or pembrolizumab plus chemotherapy vs chemotherapy alone for patients with first-line, advanced gastric cancer: the KEYNOTE-062 phase 3 randomized clinical trial. JAMA Oncol. 2020;6:1571-80.

25. Shitara K, Özgüroğlu M, Bang YJ, Bartolomeo MD, Mandalà M, Ryu $\mathrm{MH}$, et al. Pembrolizumab versus paclitaxel for previously treated, advanced gastric or gastro-oesophageal junction cancer (KEYNOTE-061): a randomised, open-label, controlled, phase 3 trial. Lancet. 2018;392:123-33.
26. Greally M, Chou JF, Chatila WK, Margolis M, Capanu M, Hechtman JF, et al. Clinical and molecular predictors of response to immune checkpoint inhibitors in patients with advanced esophagogastric cancer. Clin Cancer Res. 2019;25:6160-9.

27. Le DT, Uram JN, Wang H, Bartlett BR, Kemberling H, Eyring $\mathrm{AD}$, et al. PD-1 blockade in tumors with mismatch-repair deficiency. N Engl J Med. 2015;372:2509-20.

28. Reck M, Rodriguez-Abreu D, Robinson AG, Hui R, Csőszi T, Fülöp A, et al. Pembrolizumab versus chemotherapy for PD-L1-positive non-small-cell lung cancer. N Engl J Med. 2016;375:1823-33.

29. Carbone DP, Reck M, Paz-Ares L, Creelan B, Horn L, Steins M, et al. First-line nivolumab in stage IV or recurrent non-small-cell lung cancer. N Engl J Med. 2017;376:2415-26.

30. Overman MJ, McDermott R, Leach JL, Lonardi S, Lenz HJ, Morse MA, et al. Nivolumab in patients with metastatic DNA mismatch repair-deficient or microsatellite instability-high colorectal cancer (CheckMate 142): an open-label, multicentre, phase 2 study. Lancet Oncol. 2017;18:1182-91.

31. Haag GM, Czink E, Ahadova A, Schmidt T, Sisic L, Blank S, et al. Prognostic significance of microsatellite-instability in gastric and gastroesophageal junction cancer patients undergoing neoadjuvant chemotherapy. Int J Cancer. 2019;144:1697-703.

32. Cai Z, Rui W, Li S, Fingerhut A, Sun J, Ma J, et al. Microsatellite status affects tumor response and survival in patients undergoing neoadjuvant chemotherapy for c. Front Oncol. 2020;10:614785.

33. Smyth EC, Wotherspoon A, Peckitt C, Gonzalez D, Hulkki-Wilson S, Eltahir Z, et al. Mismatch repair deficiency, microsatellite instability, and survival: an exploratory analysis of the Medical Research Council Adjuvant Gastric Infusional Chemotherapy (MAGIC) trial. JAMA Oncol. 2017;3:1197-203.

34. Choi YY, Kim H, Shin SJ, Kim HY, Lee J, Yang HK, et al. Microsatellite instability and programmed cell death-ligand 1 expression in stage II/III gastric cancer: post hoc analysis of the CLASSIC randomized controlled study. Ann Surg. 2019;270:309-16.

35. Fuchs CS, Doi T, Jang RW, Muro K, Satoh T, Machado M, et al. Safety and efficacy of pembrolizumab monotherapy in patients with previously treated advanced gastric and gastroesophageal junction cancer: Ph. JAMA Oncol. 2018;4:e180013.

Publisher's Note Springer Nature remains neutral with regard to jurisdictional claims in published maps and institutional affiliations. 


\section{Authors and Affiliations}

\section{Hirotaka Hasegawa ${ }^{1} \cdot$ Kohei Shitara $^{2} \cdot$ Shuji Takiguchi $^{3} \cdot$ Noriaki Takiguchi $^{4} \cdot$ Seiji Ito $^{5} \cdot$ Mitsugu Kochi $^{6}$. Hidehito Horinouchi ${ }^{7}$. Takahiro Kinoshita ${ }^{8} \cdot$ Takaki Yoshikawa $^{9} \cdot K^{2}$ Muro ${ }^{10}$. Hiroyoshi Nishikawa ${ }^{11,12}$. Hideaki Suna ${ }^{13} \cdot$ Yasuhiro Kodera $^{1}[$}

1 Department of Gastroenterological Surgery, Nagoya University School of Medicine, 65 Tsurumai-cho, Showa-ku, Nagoya 466-8560, Japan

2 Department of Gastrointestinal Oncology, National Cancer Center Hospital East, Kashiwa, Japan

3 Department of Gastroenterological Surgery, Graduate School of Medical Sciences, Nagoya City University, Nagoya, Japan

4 Department of Surgery, Tsuchiura Kyodo General Hospital, Tsuchiura, Japan

5 Department of Gastroenterological Surgery, Aichi Cancer Center Hospital, Nagoya, Japan

6 Department of Digestive Surgery, Nihon University Itabashi Hospital, Tokyo, Japan

7 Department of Thoracic Oncology, National Cancer Center Hospital, Tokyo, Japan
8 Department of Gastric Surgery, National Cancer Center Hospital East, Kashiwa, Japan

9 Department of Gastric Surgery, National Cancer Center Hospital, Tokyo, Japan

10 Department of Clinical Oncology, Aichi Cancer Center Hospital, Nagoya, Japan

11 Division of Cancer Immunology, Research Institute/Exploratory Oncology Research and Clinical Trial Center, National Cancer Center, Tokyo, Japan

12 Department of Immunology, Nagoya University Graduate School of Medicine, Nagoya, Japan

13 Clinical Development Planning Division, Ono Pharmaceutical Co., Ltd., Osaka, Japan 